\title{
Static Analysis of Manipulator Components of Chiller welding fixture using Finite Element Method
}

\author{
Vaibhav V. Kulkarni*, Kaustubh Joshi, Vishwesh Kugaonkar, Prasad Honrao, Aditya Patki \\ Mechanical Engineering, MIT College of Engineering, Pune, India
}

Accepted 02 March 2016, Available online 15 March 2016, Special Issue-4 (March 2016)

\begin{abstract}
Any design process has to be followed by strength analysis to ensure safe and proper working of the component. FEA analysis can easily handle complex problems like determination of structural strength, stress, failure zones etc. In this case structural analysis of a manipulator components is carried out.
\end{abstract}

Keywords: Analysis, manipulator, FEA, automation

\section{Introduction}

Analysis is the process of breaking a complex topic or substance into smaller parts in order to gain a better understanding of it. The technique has been applied in the study of mathematics and logic since before Aristotle (384-322 B.C.), though analysis as a formal concept is a relatively recent development. Engineering analysis involves the application of scientific analytic principles and processes to reveal the properties and state of a system, device or mechanism under study. Engineering analysis is decompositional, it proceeds by separating the engineering design into the mechanisms of operation or failure, analysing or estimating each component of the operation or failure mechanism in isolation, and re-combining the components according to basic physical principles and natural laws. Structural analysis is the determination of the effects of loads on physical structures and their components.

Structures subject to this type of analysis include all that must withstand loads, such as buildings, bridges, vehicles, machinery, furniture, attire, soil strata, prostheses and biological tissue. Structural analysis incorporates the fields of applied mechanics, materials science and applied mathematics to compute a structure's deformations, internal forces, stresses, support reactions, accelerations, and stability. The results of the analysis are used to verify a structure's fitness for use, often saving physical tests. Structural analysis is thus a key part of the engineering design of structures. A structure refers to a body or system of connected parts used to support a load. A structural system is the combination of structural elements and their materials. It is important for a structural engineer to be able to classify a structure by either its form or its

*Corresponding author: Vaibhav V. Kulkarni function, by recognizing the various elements composing that structure. The structural elements guiding the systemic forces through the materials are not only such as a connecting rod, a truss, a beam, or a column, but also a cable, an arch, a cavity or channel, and even an angle, a surface structure, or a frame. Once the dimensional requirement for a structure have been defined, it becomes necessary to determine the loads the structure must support. In order to design a structure, it is therefore necessary to first specify the loads that act on it. The design loading for a structure is often specified in building codes. There are two types of codes: general building codes and design codes, engineer must satisfy all the codes requirements for a reliable structure.

There are two types of loads that structure engineering must encounter in the design. First type of load is called Dead loads that consist of the weights of the various structural members and the weights of any objects that are permanently attached to the structure.

For example, columns, beams, girders, the floor slab, roofing, walls, windows, plumbing, electrical fixtures, and other miscellaneous attachments. Second type of load is Live Loads which vary in their magnitude and location. To perform an accurate analysis a structural engineer must determine such information as structural loads, geometry, support conditions, and materials properties. The results of such an analysis typically include support reactions, stresses and displacements. This information is then compared to criteria that indicate the conditions of failure. Advanced structural analysis may examine dynamic response, stability and non-linear behavior. There are three approaches to the analysis: the mechanics of materials approach (also known as strength of materials), the elasticity theory approach (which is actually a special 
case of the more general field of continuum mechanics), and the finite element approach. The first two make use of analytical formulations which apply mostly to simple linear elastic models, lead to closed-form solutions, and can often be solved by hand. The finite element approach is actually a numerical method for solving differential equations generated by theories of mechanics such as elasticity theory and strength of materials. However, the finite-element method depends heavily on the processing power of computers and is more applicable to structures of arbitrary size and complexity.

\section{Analysis of Manipulator}

Manipulator mainly consists of a fixed holder and a moving holder. Two holders carry holding plates provided with T-slots. Moving holder can be moved longitudinally to accommodate different length chillers. For moving the moving holder the rail guides actuated by the screw drive mechanism is used. For supporting the chiller from middle section the hydraulic bed actuated by hydraulic cylinder is used. Other subassemblies consists of gear boxes, motor, bearing with holders, shafts, coupling, etc. The analysis of these components is carried out to ensure safe working of the manipulator. The analysis of all the components is shown below.

\section{Procedure}

1)The process begins with creation of CAD model of the components. Solidworks is the design and simulation software used in this case.

2)After CAD design Solidworks Simulation is used to carry out required analysis.

3) According to application type of study is sellected. For eg static, drop test,bucling, fatigue or thermal. In this application static analysis is carried out.

4) First step for static analysis is assignment of material to the components. This specifies the material properties like yeild strength and elasticity.

5)Next, fixtures and defined depending on type of contact like rolling/sliding or fixed type.

6) External loads are speciified. This can be force or torque as per the application

7) Last step is to mesh the components and evaluate the result.

8) According to requirement stress, displacement or strain analysis can be carried out.

\subsection{Fixed Holder}

The fixed holder supports the motor and gearbox assembly. A holding plate is attached to this assembly which holds the chiller. Total load on the fixed holder is $40000 \mathrm{~N}$ considering factor of safety as 2 . This load acts vertically downward.

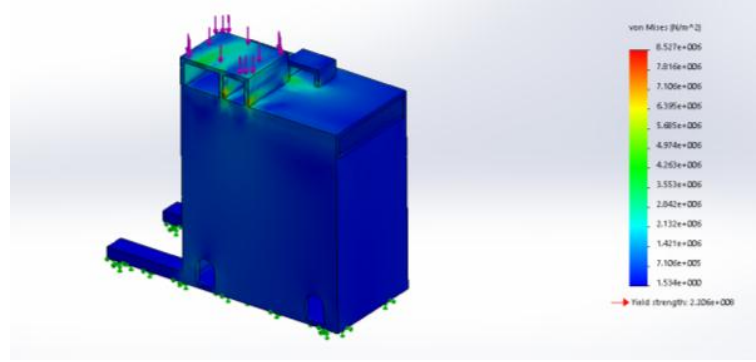

2.1.1 Stress Analysis

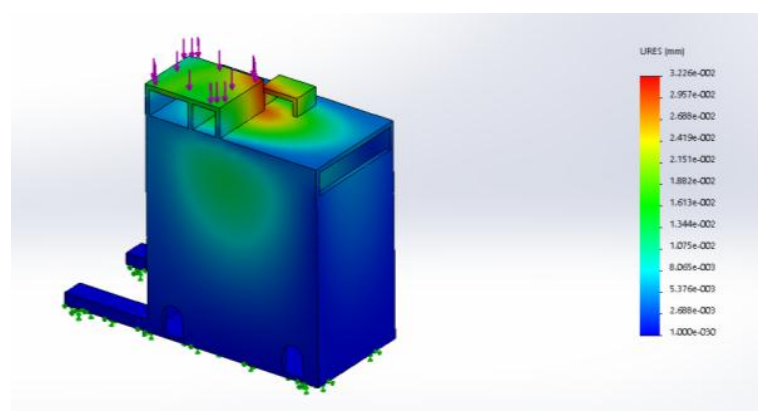

2.1.1 Displacement Analysis

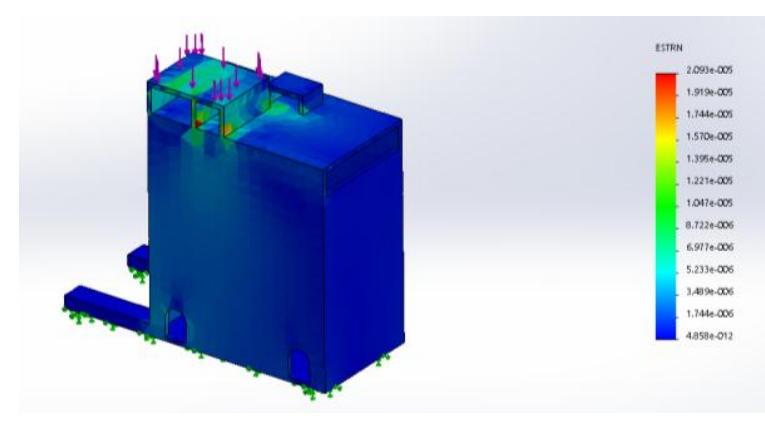

2.1.1 Strain Analysis

\subsection{Moving Holder}

This holder moves longitudinally to accommodate chillers of varying sizes. This, along with the fixed holder shares the weight of the chiller. Effectively a load of $30000 \mathrm{~N}$ acts vertically downward on this holder.

Slider contact is present at the bottom.

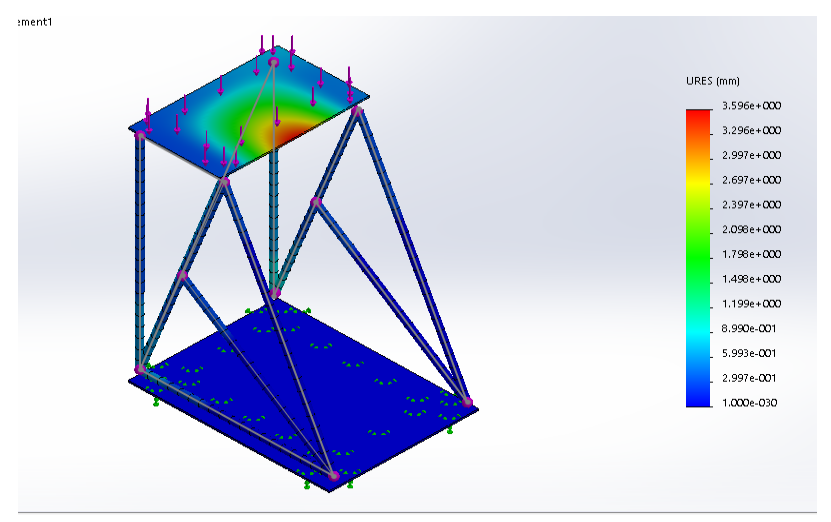

2.2.1 Displacement Analysis 

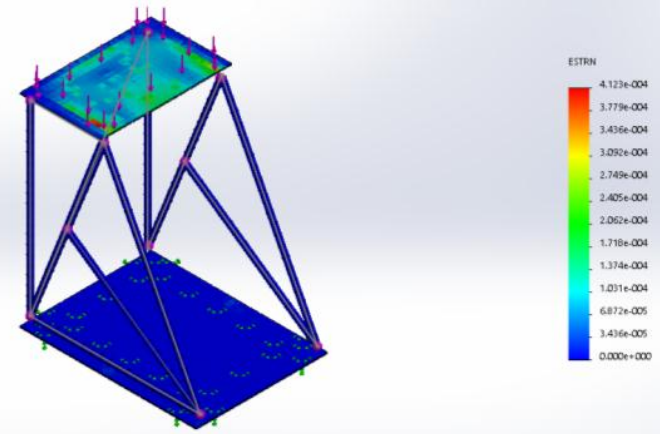

2.2.2 Strain Analysis

\subsection{Hydraulic Bed Assembly}

Hydraulic bed is provided to support the weight of the chiller. It also provides variation in height for different stages in the assembly as well as for different types of chillers. Load of $30000 \mathrm{~N}$ is applied to this bed.
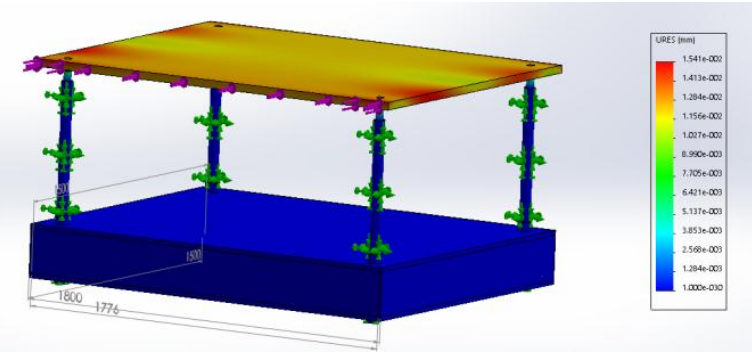

2.3 Displacement analysis

\subsection{Shaft analysis}

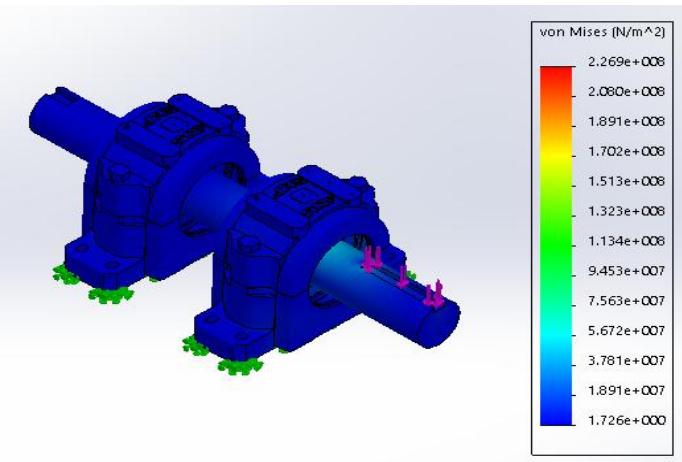

2.4.1Stress analysis

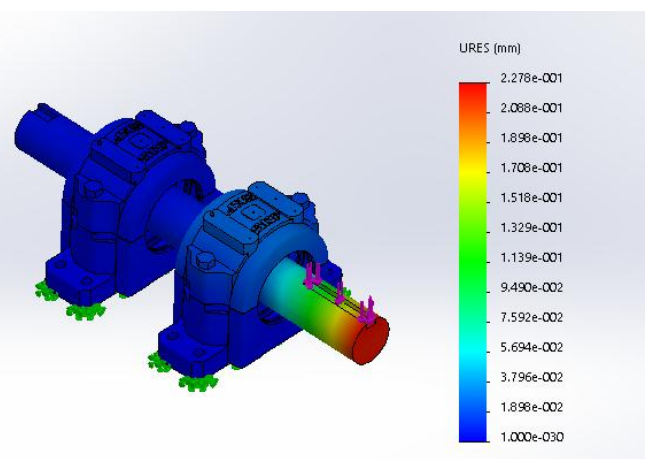

2.4.2. Displacement analysis

\subsection{Holding Plate}

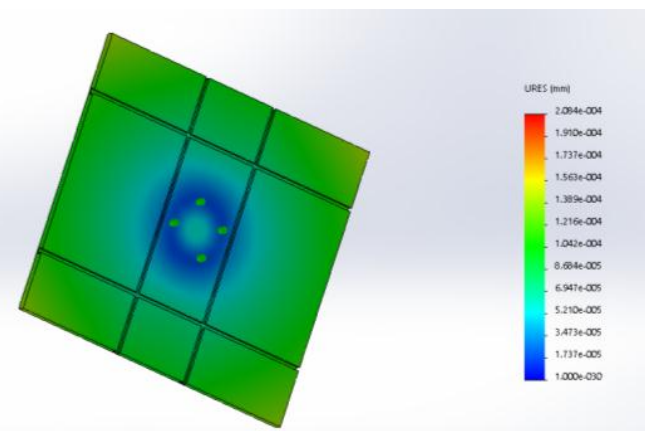

2.5.1 Strain Analysis

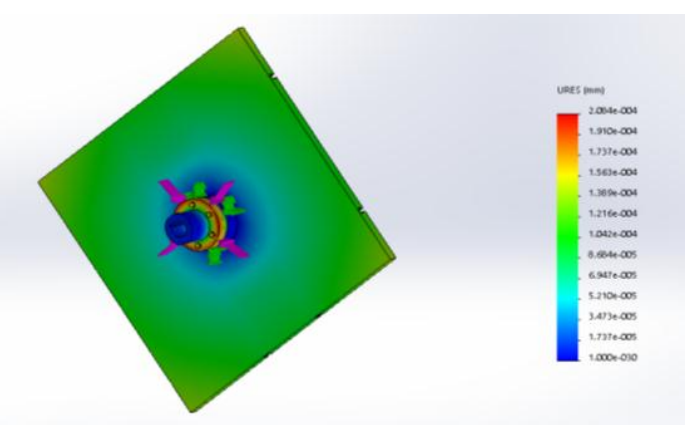

2.5.2 Strain Analysis

\section{Conclusions}

This paper has described the extensive use of finite element methods in investigating fully the structural performance of a number of building components and systems. It has demonstrated the significant benefits of using finite element tools and advanced computing facilities in obtaining safe and optimum building solutions without the need for expensive and time consuming laboratory testing. As analysis shows that all structural components are in safe working condition without any failure during working. It has demonstrated how the use of finite element tools has not only allowed the introduction of innovative and efficient building products, but also the development of accurate design methods for use by engineers, manufacturers and designers in the building.

\section{Reference}

R. Aviles, J. Vallejo, J. Aguirrebeitia, I.F. de Bustos, G. Ajuria, Optimization of linkages for generalized rigid-body guidance synthesis based on finite element models, Finite Elements in Analysis and Design 46 (2010) 721-731

Clark, S. \& Lin, Y.J. (2007). CAD tools integration for robot kinematics design assurance with case studies on PUMA robots. Industrial Robot, 34 (3), 240-248

Dwivedy, S.K. \& Eberhard, P. (2006). Dynamic analysis of flexible manipulators, a literature review. Mechanism and Machine Theory, 41, 749-777.

Karagülle, H. \& Malgaca, L. (2004). Analysis of end point vibrations of a two-link manipulator by integrated CAD/CAE procedures. Finite Elements in Analysis and Design, 40, 20492061 